\title{
VISTORIA EM OAE: TERMOGRAFIA E METODOLOGIA GDE/UNB APLICADAS A ANÁLISE DE MANIFESTAÇÕES PATOLÓGICAS
}

LIMA, JORDYSON

Estudante

Centro Universitário UDF

Brasília-DF; Brasil

jordysonnunes@gmail.com

\author{
KLANOVICHS, FERNANDO \\ Estudante \\ Centro Universitário UDF \\ Brasília-DF; Brasil \\ fernandohayne@gmail.com
}

\author{
CARVALHO, JOÃO VICTOR \\ Estudante \\ Centro Universitário UDF \\ Brasília-DF; Brasil \\ joaovitorcm.07@gmail.com
}

\author{
LIMA, HENRIQUE \\ Professor Msc. \\ Centro Universitário UDF \\ Brasília-DF; Brasil \\ hjnery@gmail.com
}

\author{
RIOS, GUTEMBERG \\ Engenheiro Mecânico \\ Abemec-DF \\ Brasília-DF; Brasil \\ abemec@abemec-df.org
}

\section{RESUMO}

Obras de Arte Especiais são construções desenvolvidas com a finalidade de transpor obstáculos, facilitando cada vez mais o acesso e transporte dos usuários. Essas estruturas estão sujeitas às ações externas devido a intemperismo ambiental que podem diminuir a sua vida útil. Por esses motivos é de suma importância realizar vistorias para constatar anomalias presentes visando o acompanhamento do estado de conservação da estrutura. Neste contexto, o presente trabalho tem como objetivo analisar e avaliar a situação física de um Viaduto localizado no encontro das quadras 11/12 no Eixo W Sul em Brasília-DF, verificando suas manifestações patológicas. Para esse fim, foi aplicado a metodologia GDE/UnB, para avaliação quantitativa do grau de deterioração dessa estrutura de concreto armado, classificando-a em função da gravidade dos problemas encontrados mediante a definição de um grau de risco, proporcionando a determinação de prioridades para ações de manuteção. Concomitantemente utilizou-se a Termografia Passiva para análise qualitativa da estrutura, a qual é uma técnica não destrutiva, em que constitui-se na percepção da radiação infravermelho emitida por um corpo através de aparelhos capacitados a detectar e assimilar esse tipo de transferência de calor. Portando após os dados coletados, com a metodologia GDE/Unb foi possível gerar o grau de deterioração da estrutura e classificar como nível alto, e juntamento com câmeras termográficas visualizou-se manifestações patológicas, como manchas de umidade, presença de lodo nos rodapés das cortinas, armaduras expostas, perda de seção do concreto, eflorescências e desplacamento do revestimento, sendo possível propor ações admissíveis que buscam suprimir os problemas em questão.

Palavras-chave: Obras de Arte Especiais, Manifestação Patológica, Manutenção.

\section{ABSTRACT}

Special Art works are constructions developed with the purpose of transpose obstacles, increasingly facilitating the access and transportation of users. These structures are subject to external actions due to environmental weathering which may shorten their useful life. For these reasons, it is extremely important to carry out surveys to find anomalies present in order to monitor the state of conservation of the structure. In this context, this paper aims to analyze and evaluate the physical situation of a viaduct located at the meeting of blocks 11/12 on the Southern Axis W in Brasilia$\mathrm{DF}$, verifying its pathological manifestations. To this end, the GDE / UnB methodology was applied for quantitative assessment of the degree of deterioration of this reinforced concrete structure, classifying it according to the severity of the problems encountered by defining a degree of risk, providing the determination of priorities for this purpose maintenance actions. Concomitantly, Passive Thermography was used for qualitative analysis of the structure, which is a non-destructive technique, which consists in the perception of infrared radiation emitted by a body through devices capable of detecting and assimilating this type of heat transfer. . Following the data collected, with the GDE / Unb methodology it was possible to generate a degree of deterioration of the structure and rate as high level, and coupling with thermographic cameras visualized pathological manifestations, such as moisture stains, presence of sludge on the curtain walls, exposed reinforcement, loss concrete section, efflorescences and coating peeling, and it is possible to propose admissible actions that seek to suppress the problems in question.

Keywords: Special Art works, Pathological Manifestations, Maintenance. 


\section{INTRODUÇÃO}

As estruturas de concreto não possuem vida útil ilimitada, devido estarem expostas a intempirismo ambiental. Hoje no Brasil os estudos sobre deterioração da estrutura ganham cada vez mais destaque, e com a necessidade desenvolveu uma área da engenharia civil chamada de "Patologia das construções" a qual é o campo responsável por estudar origens, formas de manifestação, consequências e degradação das estruturas. A patologia é muito mais que reconhecer anomalias em uma estrutura, é sobre o projeto e sua concepção estrutural e em sua base sobre a formação do engenheiro civil, já que a vida útil se tornou um dado fundamental para a concepção de projetos (SOUSA E RIPPER, 1988).

Como ocorre em todas as estruturas existentes, os materiais constituintes das Obras de Arte Especiais (OAEs) se degradam com o tempo, implicando em alterações nas suas propriedades e no seu desempenho, podendo culminar em uma situação onde a estrutura não seja mais compatível com as condições consideradas na fase de projeto. Caso não haja uma intervenção adequada no prazo correto, a estrutura atingirá uma situação em que a limitação do carregamento ou a sua completa interdição serão as únicas alternativas (VERLY, 2015).

Segundo Vitório (2006) a falta de uma cultura de manutenção, em especial a preventiva, faz com que os órgãos responsáveis pelas obras públicas, nos níveis federal, estadual e municipal, priorizem apenas a execução, não havendo maiores preocupações com as questões relacionadas à conservação. Isto pode ser constatado através da simples observação das obras de infraestrutura, especialmente as pontes e viadutos, conhecidas como Obras de Arte Especiais.

A necessidade de cuidar das Obras de Arte Especiais já construídas é dada em âmbito nacional. Pois, de acordo com Fontes (2007), a eficiência da malha rodoviária é fundamental em um país que utiliza o sistema rodoviário como seu sistema principal de transporte. A fim de se ter um controle sobre a situação das OAEs já construídas, faz-se necessário acompanhá-las por meio de inspeções.

Com a evolução do conhecimento concluiu-se que a vida útil da estrutura depende, substancialmente, de níveis adequados de manutenção, principalmente porque os eventuais problemas estruturais, sendo descobertos em seu início, teriam seus efeitos minorados e os custos de reparo seriam reduzidos. Entretanto, embora seja crescente o reconhecimento da importância da manutenção estrutural, são ainda insuficientes, mesmo em países desenvolvidos, as disposições normativas específicas para programas de manutenção. Em geral, as normas têm dedicado grande atenção às disposições de projeto e execução tendo a durabilidade como requisito essencial sem, no entanto, estabelecer critérios objetivos de manutenção (KRAUS, 1994).

Para a determinação das intervenções adequadas em obras de arte especiais e os prazos corretos, podem ser utilizados sistemas de gestão de OAEs. Esses sistemas podem ser mais simples, compostos apenas por um banco de dados, ou mais completos, que utilizam modelos de deterioração para avaliar a situação futura da estrutura e determinar a melhor possibilidade de alocação de recursos. Assim, um sistema de gestão de OAEs é uma ferramenta efetiva de alocação de recursos limitados em atividades relacionadas à OAEs (AASHTO, 2013; SMITH e SILVA FILHO, 1999). E os dados que constituem os sistemas de gestão dessas estruturas podem ser realizados de acordo com diferentes metodologias.

Devido a esses fatores, foi realizada vistoria em um viaduto localizado entre as tesourinhas do eixo L e W, das quadras 11/12 da Asa Sul, Brasília-DF. Inicialmente utilizou a técnica da Termográfica, a qual é umas das técnicas de ensaios não-destrutivos, ou seja, vistoriam materiais ou equipamentos sem a necessidade de danificá-los. Esse método constituise na percepção da radiação infravermelho emitida por um corpo através de um aparelho capacitado a detectar e assimilar esse tipo de transferência de calor (MEDEIROS, 2010). Na vistoria aplicou-se a Termografia passiva, em que é aquela que se dispõem do sol como a fonte de transferência de calor.

Posteriormente para análise do grau de deterioração da estrutura utilizou-se a metodologia GDE/UnB, a qual avalia quantitativamente as estruturas de concreto. Essa metodologia é adequada para análise das manifestações patológicas, pois é possível obter de forma rápida e objetiva, por meio dos dados coletados em campo, aplicados em formulações o nível de deterioração e as ações a serem adotadas na estrutura estudada.

\subsection{Objetivos}

O presente trabalho tem como objetivo analisar e avaliar a situação física de um Viaduto localizado no encontro das quadras 11/12 entre o Eixo W Sul em Brasília-DF, verificando suas manifestações patológicas. Utilizando a metodologia GDE/UnB, para avaliação quantitativa do grau de deterioração dessa estrutura de concreto armado, classificando-a em função da gravidade dos problemas encontrados mediante a definição de um grau de risco, 
proporcionando a determinação de prioridades para ações de manutenção, e como auxílio utilizar câmeras termográficas para detectar manifestações patológicas ocultas nessa estrutura, como presença de umidade.

\section{METODOLOGIA}

\subsection{Metodologia GDE/Unb}

A metodologia GDE foi elaborada pelo Programa de Pós-Graduação em Estruturas e Construção Civil da Universidade de Brasília (PECC-UnB) a qual permite realizar a avaliação quantitativa do grau de deterioração de estruturas de concreto, analisando e quantificando as manifestações dos danos e suas evoluções, assim, visa dar criticidade as peças estruturais de uma edificação, viabilizando a definição de prioridades para realização das manutenções. Verly (2015) aplicou a metodologia em estruturas de viadutos com as devidas alterações específicas, além disso, ele também criou o Manual de Aplicação da Metodologia GDE/UnB a Obras de Arte Especiais, propondo modificações na formulação do grau de deterioração da estrutura (Gd).

Segue o fluxograma utilizado, conforme figura 1, as quais contem às etapas que foram realizadas de forma sequencial e sistemática, em que foi desenvolvido por CASTRO (1994).

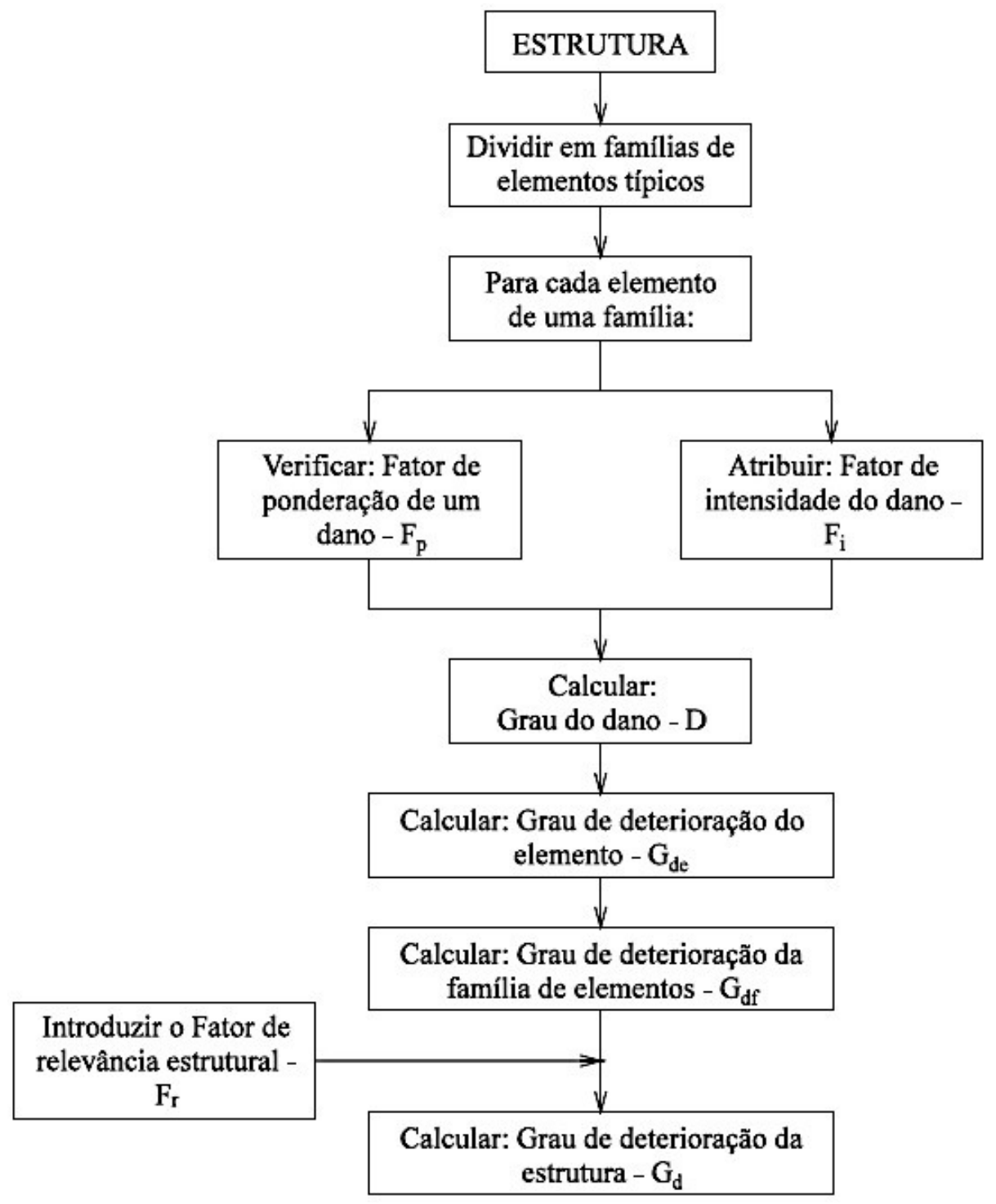

Figura 1: Fluxograma da metodologia GDE/UnB (CASTRO, 1994) 


\subsubsection{Divisão em família de elementos típicos}

Nesta etapa agrupou-se os elementos de acordo com suas características de funcionalidades estruturais. Dessa forma foram definidas as famílias a seguir: lajes, guarda-corpos, cortinas, alas, juntas de dilatação e pista de rolamento.

\subsubsection{Fator de ponderação do dano (Fp)}

Esse fator tem como finalidade quantificar a importância relativa de um determinado dano no que se refere às condições de segurança, funcionalidade e estética dos elementos de uma determinada família (CASTRO 1994), podendo variar de zero a cinco. Através das tabelas 1 a 5 abaixo, obteve-se os valores referentes aos danos das manifestações patológicas relacionadas ao desempenho operacional.

Tabela 1 - Família de elemento estrutural, dano e fator de ponderação (Fp) em Lajes (VERLY, 2015)

\begin{tabular}{|c|c|}
\hline \multicolumn{2}{|c|}{ Lajes } \\
\hline Danos & Fp \\
\hline Carbonatação do concreto & 3 \\
Cobrimento deficiente & 3 \\
Contaminação por cloretos & 3 \\
Corrosão de armaduras & 5 \\
Desagregação & 3 \\
Desplacamento & 3 \\
Eflorescências & 2 \\
Falha de concretagem & 2 \\
Fissuras & 2 a $5 *$ \\
Flechas & 5 \\
Infiltração de água & 3 \\
Manchas & 3 \\
\hline
\end{tabular}

Tabela 2 - Família de elemento estrutural, dano e fator de ponderação (Fp) nos Guarda-corpos (VERLY, 2015)

\begin{tabular}{|c|c|}
\hline \multicolumn{2}{|c|}{ Guarda-corpos } \\
\hline Danos & Fp \\
\hline Carbonatação do concreto & 3 \\
Cobrimento deficiente & 3 \\
Contaminação por cloretos & 4 \\
Corrosão de armaduras & 5 \\
Desagregação & 3 \\
Desplacamento & 3 \\
Eflorescências & 2 \\
Falha de concretagem & 2 \\
Fissuras & 2 a $5^{*}$ \\
Manchas & 3 \\
Sinais de esmagamento & 4 \\
\hline
\end{tabular}


Tabela 3 - Família de elemento estrutural, dano e fator de ponderação (Fp) nas Cortinas/Alas (VERLY, 2015)

\begin{tabular}{|c|c|}
\hline \multicolumn{2}{|c|}{ Cortina/alas } \\
\hline Danos & Fp \\
\hline Carbonatação do concreto & 3 \\
Cobrimento deficiente & 3 \\
Contaminação por cloretos & 4 \\
Corrosão de armaduras & 5 \\
Desagregação & 3 \\
Deslocamento por empuxo & 5 \\
Desplacamento & 3 \\
Desvio de geometria & 3 \\
Eflorescências & 2 \\
Falha de concretagem & 2 \\
Fissuras & 2 a $5^{*}$ \\
Infiltração de água & 3 \\
Manchas & 3 \\
Sinais de esmagamento & 5 \\
\hline
\end{tabular}

Tabela 4 - Família de elemento estrutural, dano e fator de ponderação (Fp) nas Juntas de dilatação (VERLY, 2015)

\begin{tabular}{|c|c|}
\hline \multicolumn{2}{|c|}{ Juntas de dilatação } \\
\hline Danos & Fp \\
\hline $\begin{array}{c}\text { Obstrução de junta } \\
\text { Desgaste do material de } \\
\text { preenchimento da junta } \\
\text { Umidade }\end{array}$ & 5 \\
\end{tabular}

Tabela 5 - de elemento estrutural, dano e fator de ponderação (Fp) na Pista de rolamento (VERLY, 2015)

\begin{tabular}{|c|c|}
\hline \multicolumn{2}{|c|}{ Pista de rolamento } \\
\hline Danos & Fp \\
\hline Descontinuidade & 5 \\
Desgaste superficial & 5 \\
Desgaste da sinalização & 5 \\
\hline
\end{tabular}

\subsubsection{Fator de intensidade do dano (Fi)}

O fator de intensidade (Fi) não leva em consideração o elemento estrutural no qual dano se encontra e varia de zero a quatro. O Fi tem por objetivo classificar a gravidade e a evolução isolada do dano em um determinado elemento (VERLY, 2015), conforme a Tabela 6.

Tabela 6 - Classificação de lesóes de determinado elemento (FONSECA, 2007)

\begin{tabular}{|c|c|}
\hline Classificação & Fator de Intensidade (Fi) \\
\hline Sem lesões & 0 \\
Lesões leves & 1 \\
Lesões toleráveis & 2 \\
Lesões graves & 3 \\
Estado crítico & 4 \\
\hline
\end{tabular}




\subsubsection{Grau do dano (D)}

O grau de dano é calculado através do fator de intensidade e de ponderação, nos elementos estruturais, que leva em consideração o mecanismo de corrosão em armaduras de reforço de concreto, em que este modelo pode ser aplicado mais largamente para descrever a deterioração em geral no interior de uma estrutura de concreto, a qual foi proposto por Tuutti em 1982, e generalizada pelo Código Modelo MC - 90 CEB-FIP em 1991. Segue as formulações para encontrar o grau do dano, demonstrado nas equações 1 e 2.

$$
\begin{array}{r}
D=0,8 \cdot F_{i} \cdot F_{p} \text { para } F_{i} \leq 2,0 \\
D=\left(12 \cdot F_{i}-28\right) \cdot F_{p} \text { para } F_{i} \geq 3,0
\end{array}
$$

Onde:

Fi: Fator de Intensidade;

Fp: Fator de Ponderação.

\subsubsection{Grau de deterioração do elemento (Gde)}

Obtendo os graus isolados de cada dano, é possível calcular o grau de deterioração do elemento através da equação 3 , proposto por Lopes (1988).

$$
G_{d e}=D_{\text {máx }}\left[1+\frac{\sum_{i=1}^{n} D_{(i)}-D_{\text {máx }}}{\sum_{i=1}^{n} D_{(i)}}\right]
$$

Onde:

Gde: Grau de deterioração do elemento;

Di: Grau do dano de índice "i”;

Dmáx: Maior Grau do dano encontrado no elemento;

$\mathrm{n}$ : Número de danos encontrados no elemento.

Após encontrar o valor calculado de Gde para cada elemento, algumas ações são recomendadas de acordo com o nível de deterioração, podendo ser adotadas, para que a estrutura permaneça segura e com uma vida útil prolongada, como pode se observar na tabela 7 .

Tabela 7 - Classificação dos níveis de deterioração do elemento pela metodologia GDE/UnB (FONSECA, 2007)

\begin{tabular}{|c|c|c|}
\hline Nível de Deterioração & Gde & Ações a serem tomadas \\
\hline Baixo & $0-15$ & $\begin{array}{r}\text { Estado aceitável. Manutenção Preventiva. } \\
\text { Médio }\end{array}$ \\
$15-50$ & $\begin{array}{r}\text { Definir prazo/natureza para nova inspeção. Planejar intervenção em longo } \\
\text { prazo (máximo } 2 \text { anos). }\end{array}$ \\
Alto & $50-80$ & $\begin{array}{r}\text { Definir prazo/natureza para inspeção especializada detalhada. Planejar } \\
\text { intervenção em médio prazo (máximo 1 ano). }\end{array}$ \\
Sofrível & $80-100$ & $\begin{array}{r}\text { Definir prazo/natureza para inspeção especializada detalhada. Planejar } \\
\text { intervenção em curto prazo (máximo 6 meses). }\end{array}$ \\
Crítico & $>100$ & Inspeção especial emergencial. Planejar intervenção imediata. \\
\hline
\end{tabular}




\subsubsection{Grau de deterioração de uma família de elementos (Gdf)}

O Gdf tem como objetivo avaliar o conjunto de elementos pertencentes a uma mesma família (VERLY, 2015). Para calcular esse grau de deterioração de uma família de elementos, conforme equação 4, pegou-se os valores obtidos apenas dos elementos com nível de deterioração médio ou superior, ou seja, com Gde $\geq 15$.

$$
G_{d f}=G_{d e m a ́ x}\left[\sqrt{1+\frac{\sum_{i=1}^{n} G_{d e(i)}-G_{d e m a ́ x}}{\sum_{i=1}^{n} G_{d e(i)}}}\right]
$$

Onde:

Gdf: Grau de deterioração de uma família de elementos;

Gde máx: Grau de deterioração máxima do elemento;

Gde(i): Grau de deterioração do elemento de ordem (i);

\subsubsection{Fator de relevância estrutural (Fr)}

Segundo Moreira (2007) o fator de relevância tem como objetivo, considerar a importância relativa de uma família de elementos quanto ao comportamento e desempenho da estrutura como um todo. Fonseca (2007) utilizou os valores de Fr descritos na tabela 8 .

Tabela 8 - Fatores de Relevância Estrutural (FONSECA, 2007)

\begin{tabular}{|c|c|}
\hline Famílias em Conjunto & $\begin{array}{c}\text { Fatores de Relevância } \\
\text { Estrutural (Fr) }\end{array}$ \\
\hline Elementos de composição arquitetônica. & 1,0 \\
Reservatório superior. & 2,0 \\
Escadas/rampas, reservatório inferior, cortinas, lajes secundárias, \\
juntas de dilatação. \\
Lajes, fundações, vigas secundárias, pilares secundários. \\
Vigas e pilares principais.
\end{tabular}

\subsubsection{Grau de deterioração da estrutura $(\mathrm{Gd})$}

Para obter o grau de deterioração da estrutura, é necessário considerar o fator de relevância de cada família, conforme tabela 8, e o grau de deterioração da família. Segue a equação 5 utilizada para encontrar o Gd. E após a aplicação dessa equação o grau pode ser classificado em níveis, conforme tabela 9.

$$
G_{d}=\frac{\sum_{i=1}^{k} F_{r(i)} \cdot G_{d f(i)}}{\sum F_{r(i)}}
$$

Onde:

k: Número de famílias da estrutura;

Fr: Fatores de relevância estrutural;

Gdf: Grau de deterioração de uma família de elementos;

Gd: Grau de deterioração global da estrutura. 
Tabela 9 - Classificação do nível de deterioração da estrutura (FONSECA, 2007)

\begin{tabular}{|c|c|c|}
\hline Nível de Deterioração & Gde & Ações a serem tomadas \\
\hline Baixo & $0-15$ & $\begin{array}{c}\text { Estado aceitável. Manutenção Preventiva. } \\
\text { Médio }\end{array}$ \\
Alto & $15-50$ & $\begin{array}{r}\text { Definir prazo/natureza para nova inspeção. Planejar intervenção em } \\
\text { longo prazo (máximo 2 anos). }\end{array}$ \\
Sofrível & $80-80$ & $\begin{array}{r}\text { Definir prazo/natureza para inspeção especializada detalhada. Planejar } \\
\text { intervenção em médio prazo (máximo 1 ano). } \\
\text { Crítico }\end{array}$ \\
\hline
\end{tabular}

\subsection{Aplicação da metodologia GDE/Unb na OAE}

O viaduto em estudo é de concreto armado e está localizado entre as quadras 111/112 no eixo W na Asa Sul em Brasília-DF. Segue figura 2 representativa do local.

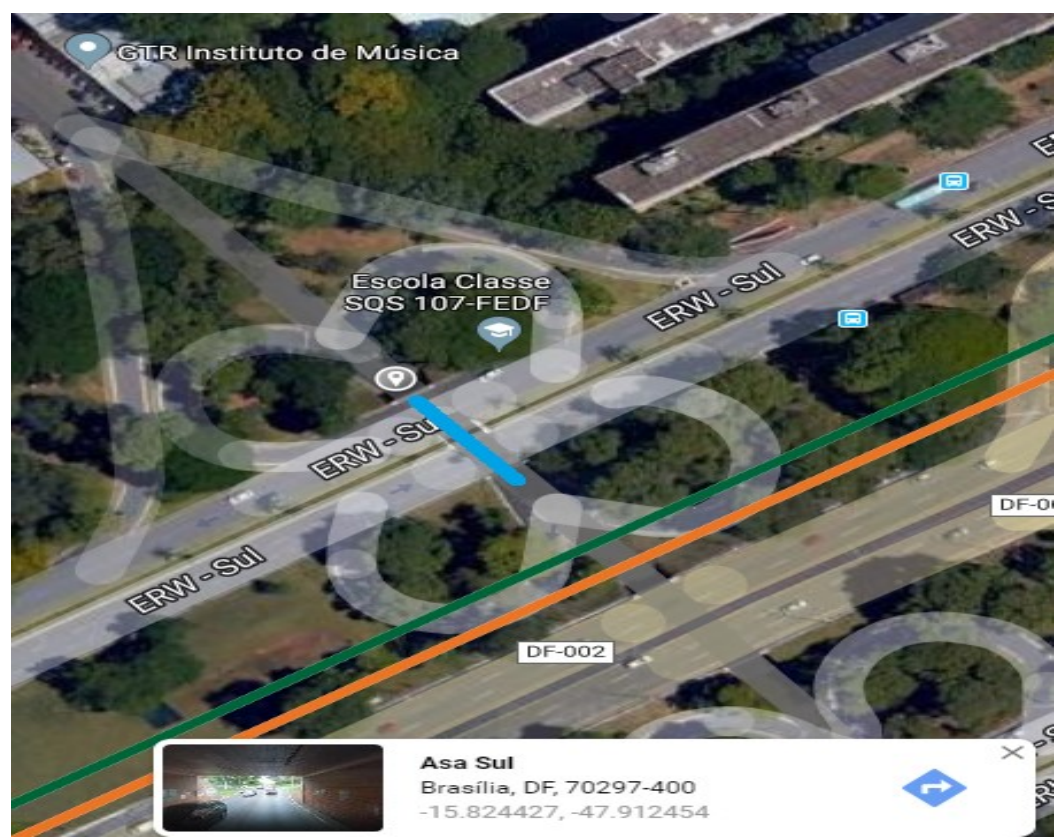

Figura 2: Localização do viaduto analisado (Google Maps, 17/12/2019)

A aplicação do método GDE/UnB, foi realizada mediante avaliações e inspeções in loco, utilizando as adequações necessárias na divisão dos elementos das OAEs em famílias, de acordo com Euqueres (2011).

\subsection{Aplicação da Termografia Passiva na OAE}

Com as imagens termográficas é possível identificar algumas anomalias como, fissuras e regiões com umidade, além de ser possível a identificação de heterogeneidades superficiais. Coleta-se essas imagens com uso de uma câmera especial, e podem ser feitas a distâncias significativas, sendo até vinte metros com equipamentos usuais, de acordo com Gomes e Xavier (2018).

Neste estudo foi realizado uma análise qualitativa aplicando a técnica da termografia por infra-vermelho, identificando possíveis manifestações patológicas e não conformidades encontradas no viaduto. Utilizou-se a câmera de infravermelho Fluke TiS60. Segue suas características na tabela 10. 
Tabela 10 - Especificação da câmera termográfica Fluke TiS60

\begin{tabular}{|c|c|c|c|c|c|}
\hline \multicolumn{7}{|c|}{ Fluke TiS60 } \\
\hline Dimensões & Precisão & $\begin{array}{c}\text { Faixa dinâmica } \\
\text { de cena }\end{array}$ & $\begin{array}{c}\text { Captura de } \\
\text { imagem }\end{array}$ & $\begin{array}{c}\text { Resolução } \\
\text { térmica }\end{array}$ & Emissividade \\
\hline $267 \times 101 \times 145 \mathrm{~mm}$ & $\pm 2{ }^{\circ} \mathrm{C}$ ou $2 \%$ &.$-20^{\circ} \mathrm{C}$ a $550^{\circ} \mathrm{C}$ & Câmera $5 \mathrm{MP}$ & $260 \times 195$ & 0,92 \\
\hline
\end{tabular}

\section{RESULTADOS}

Com o uso de manuais, informações técnicas, vistorias, termografia e os procedimentos recomendados pela metodologia GDE/UnB descritos anteriormente, foi caracterizado o estado de deterioração do viaduto localizado no Eixo W Sul, Brasília-DF. Segundo Euqueres (2011), os valores de Gde abaixo de 15 favorecem uma avaliação mais adequada da estrutura, sendo que a não utilização desses podem gerar falhas na priorização da manutenção. Esse trabalho seguiu essas recomendações por Euqueres (2011).

Nas vistorias com o auxílio de câmeras termográficas observou-se na estrutura do viaduto algumas manifestações patológicas que afetam o desempenho e a funcionalidade da estrutura de concreto, sendo elas, carbonatação do concreto, cobrimento deficiente nos guarda-corpos, cortinas e lajes, corrosão de armaduras na cortina, desagregação, desplacamento, eflorescências, fissuras, manchas e presença de vegetação devido a infiltração de água, nas figuras 3 e 4 e nos anexos é possível visualizar algumas dessas manifestações atuando na estrutura. Devido o viaduto analisado ter revestimento cerâmico (gressite), com o uso de câmeras termográficas foi possível quantificar de forma mais precisa alguns fatores necessários para gerar o grau de deterioração, logo a técnica da termografia complementou a metodologia GDE/UnB.

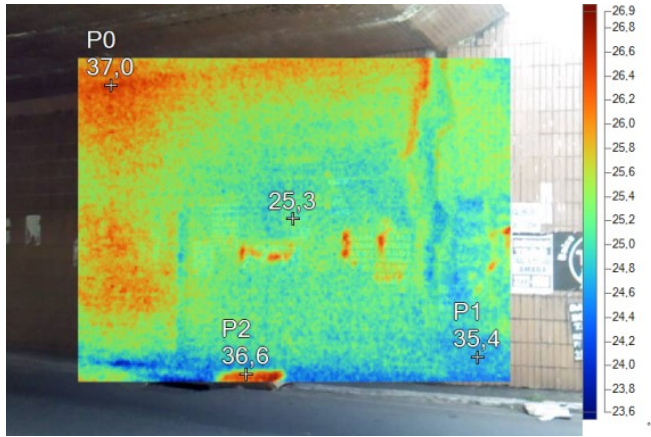

Figura 3 - Termograma da Cortina

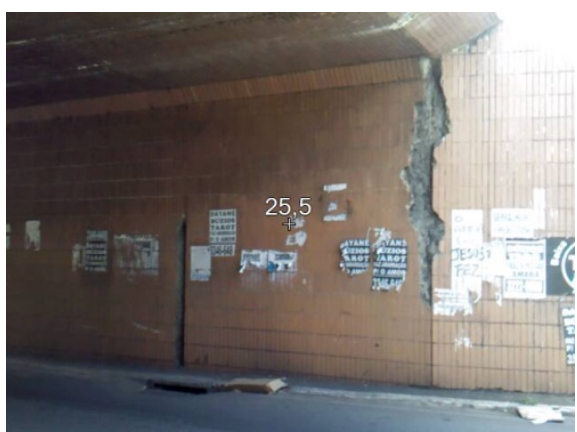

Figura 4 - Presença de umidade na Cortina

Posteriormente realizando as análises constatou-se que o viaduto obtve Gd igual a 52,92, que segundo a metodologia o nível de deterioração pode ser classificado como alto. Seguem as tabelas 11 e 12 com os resultados obtidos.

Tabela 11 - Resultado do cálculo do Gde e Gdf pela metodologia GDE/UnB

\begin{tabular}{|c|c|c|c|}
\hline Elementos & Gde & Gdf & Fr \\
\hline Cortinas e Alas & 71,03 & 87,00 & 4 \\
Guarda Corpo & 69,19 & 84,74 & 1 \\
Lajes & 9,01 & 11,04 & 4 \\
Juntas de dilatação & 61,82 & 75,71 & 2 \\
Pista de rolamento & 5,50 & 6,74 & 1 \\
\hline
\end{tabular}

Tabela 12 - Resultado do cálculo do Gd, nível de deterioração e ações recomendadas pela metodologia GDE/UnB

\begin{tabular}{|c|c|c|}
\hline Gd & Nível de deterioração & Ações recomentadas \\
\hline 52,92 & Alto & Definir prazo para inspeção especializada. Planejar intervenção em \\
médio prazo (máximo 1 ano).
\end{tabular}




\section{CONCLUSÃO}

A metodologia GDE/UnB mostrou resultados satisfatórios na aplicação, a qual foi possível identificar o nível de gravidade das lesões e recomendar ações, sendo possível planejar intervenções de acordo com o grau de deterioração da estrutura, desta forma, essa metodologia pode ser considerada adequada para avaliação de diversos tipos de estruturas de concreto armado. As inspeções visuais realizadas in loco possibilitaram identificar manifestações patológicas como, exposição e corrosão das armaduras, desagregação e desplacamento do concreto, eflorescências e entre outros, e os elementos que apresentam o maior nível de degradação devido a essas anomalias são as cortinas, alas e guarda-corpo.

Com o auxílio da termografia foi possível gerar resultados mais precisos, pois geralmente nas vistorias de algumas estruturas não é possível visualizar infiltrações de águas sem realizar extração de material, ou ensaios mais específicos, assim sendo, durante a determinação do fator de intensidade - Fi referente a machas de umidade e infiltrações de água nos elementos estruturais, obteve segurança nos dados coletados. Portanto a compatibilização do uso da técnica da termografia e a metodologia GDE/UnB se torna viável para análise de viadutos.

\section{AGRADECIMENTOS}

Agradecemos ao grupo TERMODAN, pela oportunidade de participação nesse projeto multidisciplinar, que nos auxiliou na aplicação da técnica da termografia no viaduto analisado. Agradecemos ao Centro Universitário UDF pelo apoio financeiro, o qual se tornou possível à apresentação deste artigo no congresso.

\section{REFERÊNCIAS}

ASSOCIAÇÃO BRASILEIRA DE NORMAS TÉCNICAS- ABNT. NBR 6118: Projeto de estruturas de concretoProcedimento. Rio de Janeiro, RJ. 2014.

ASSOCIAÇÃO BRASILEIRA DE NORMAS TÉCNICAS - ABNT, NBR 9452: Vistoria de pontes e viadutos de concreto. Rio de Janeiro, 2016, 11 p.

FONSECA, R. P. A estrutura do Instituto Central de Ciências: Aspectos históricos, científicos e tecnológicos de projeto, execução, intervenções e propostas de manutenção. Dissertação de Mestrado em Estruturas e Construção Civil, Departamento de Engenharia Civil e Ambiental, Universidade de Brasília, Brasília, DF, 2007.

GOMES, Marcos. XAVIER, Thássio. Levantamento e cadastramento de patologias estruturais utilizando fotografias termográficas e drones na composição de banco geográficos. Pesquisa de iniciação cientifica. Centro Universitário de Brasília UNICEUB. Brasília, DF. 2018.

LOPES, B.A.R., Sistema de manutenção estrutural para grandes estoques de edificações: Estudo para a inclusão do componente Estrutura de Concreto. Dissertação (Mestrado) - Universidade de Brasília, Brasília, DF,1998, 308p.

SOUZA, V. C.; RIPPER, T. Patologia, recuperação e reforço de estruturas de concreto. São Paulo: Pini, 1998. 255 p.

VERLY, Rogério. Avaliação de metodologias de inspeção como instrumento de priorização de intervenções em obras de artes especiais. Dissertação de mestrado em estruturas e construção civil. Universidade de Brasília. Brasília, DF. 2015.

VITÓRIO, J. A. P. Vistorias, Conservação e Gestão de Pontes e Viadutos de Concreto. Anais do $48^{\circ}$ Congresso Brasileiro do Concreto, 2006. 


\section{ANEXO I - FOTOS DO VIADUTO, TERMOGRAFIA E DIGITAL}

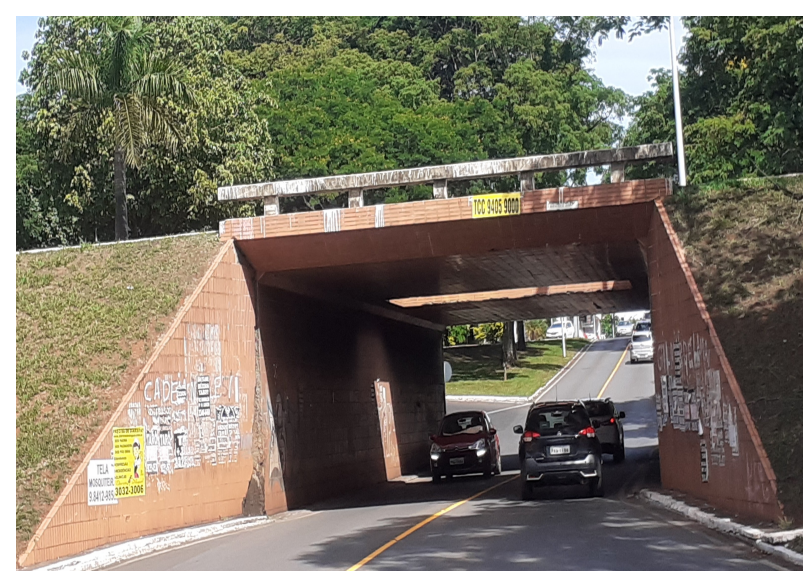

Figura 5 - Vista frontal do viaduto

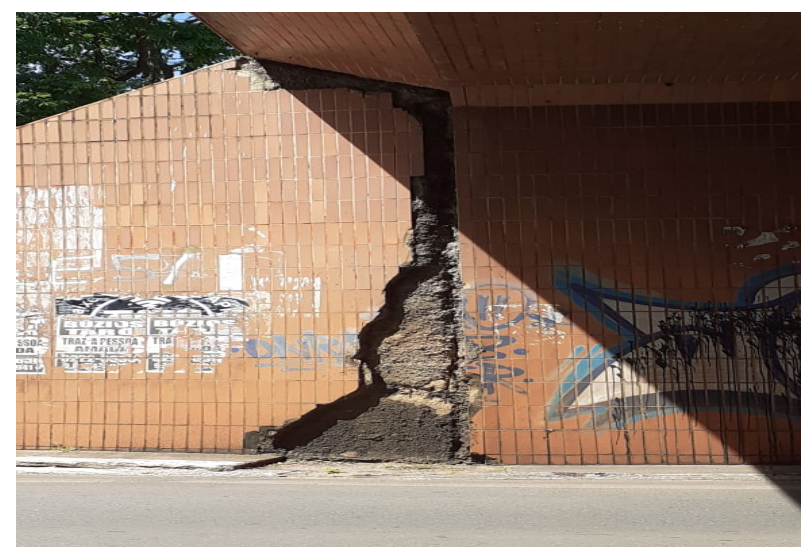

Figura 7 - Umidade e armadura exposta na ala

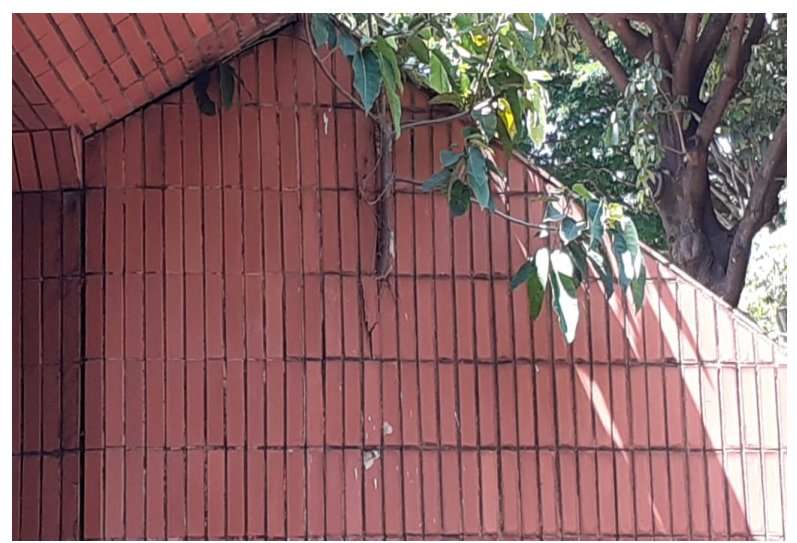

Figura 9 - Presença de vegetação na ala

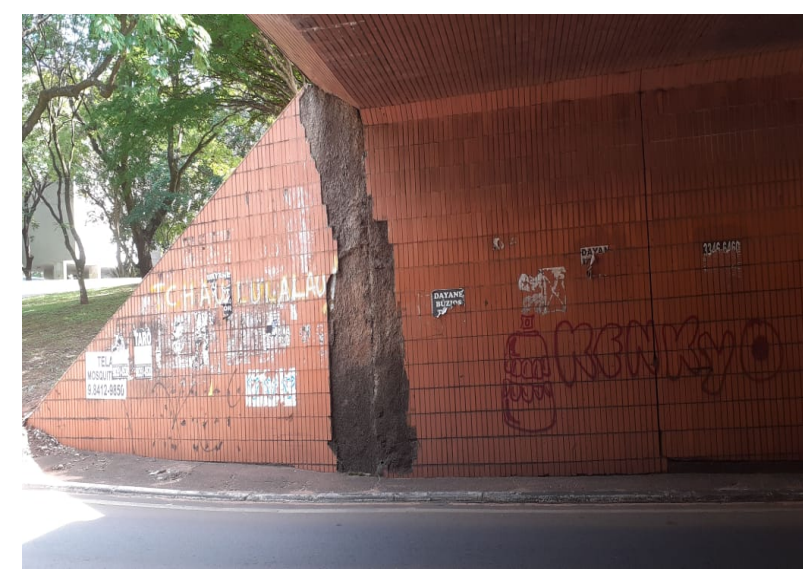

Figura 6 - Desplacamento do revestimento Ala/Cortina

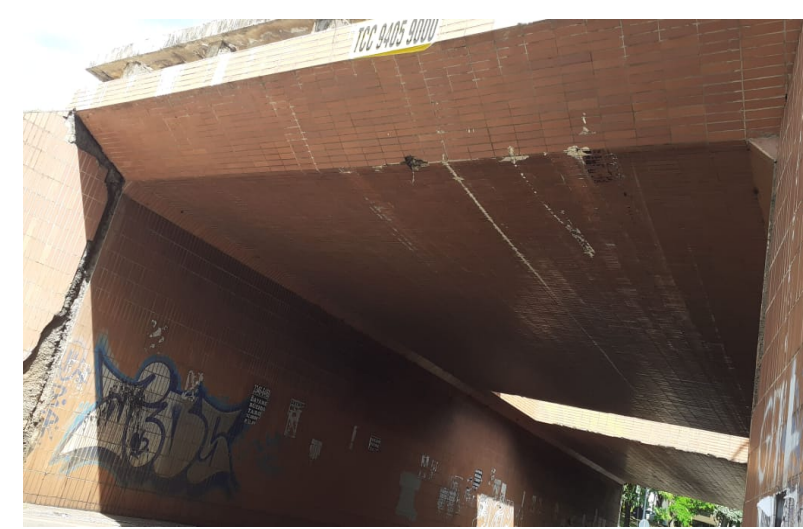

Figura 8 - Choque mêcanico na laje

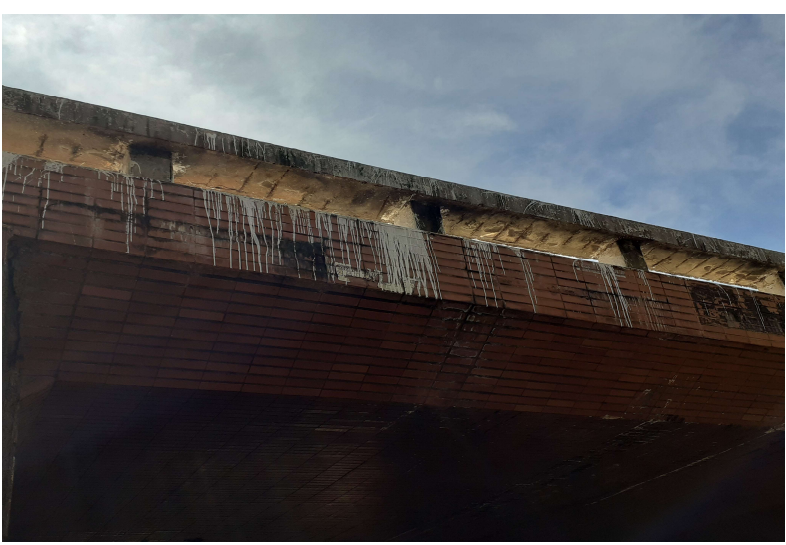

Figura 10 - Armadura exposta no guarda-corpo 


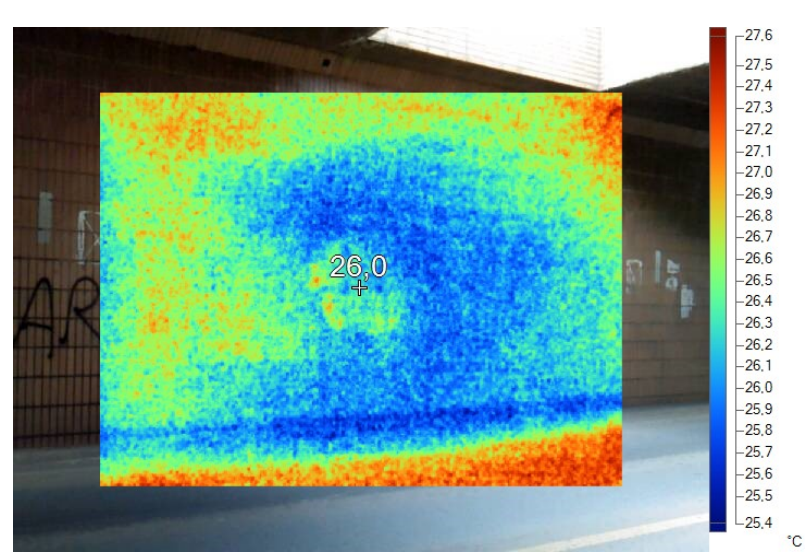

Figura 11 - Termograma da cortina

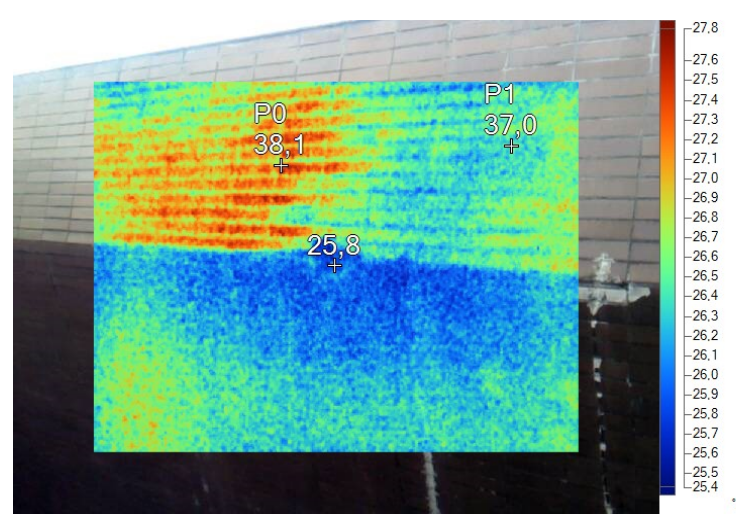

Figura 13 - Termograma da laje tabuleiro

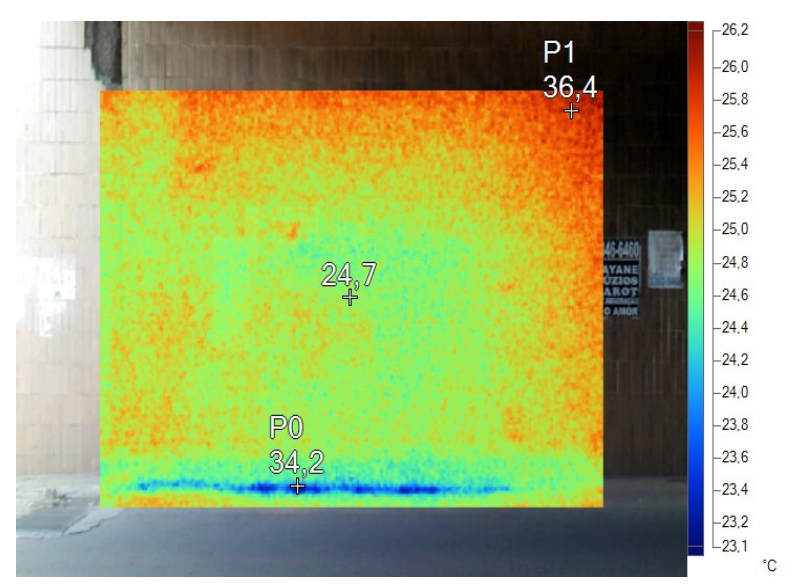

Figura 35 - Termograma rodapé da cortina

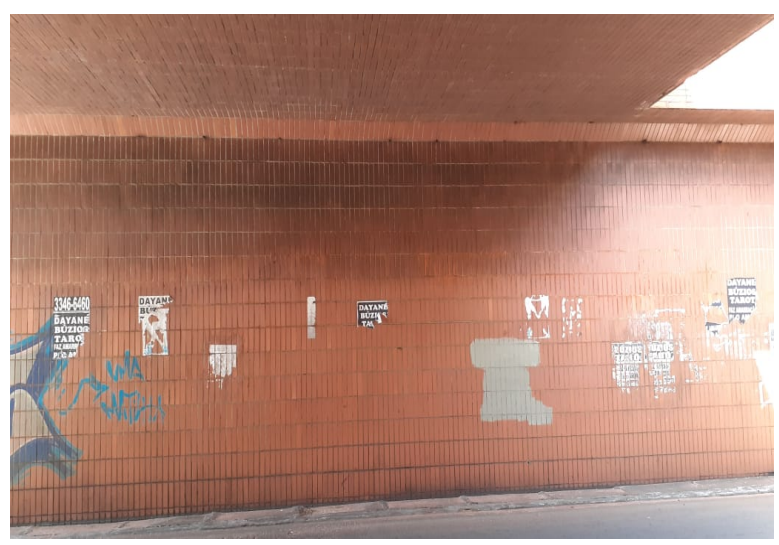

Figura 12 - Foto digital da cortina

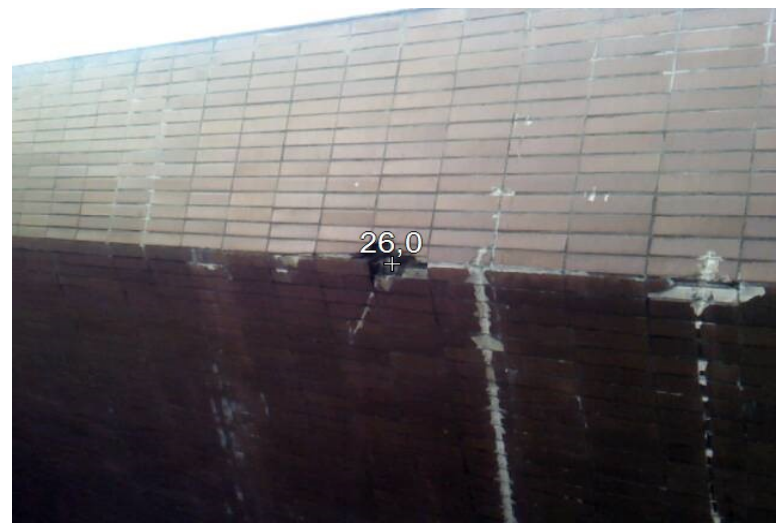

Figura 14 - Foto digital da laje tabuleiro

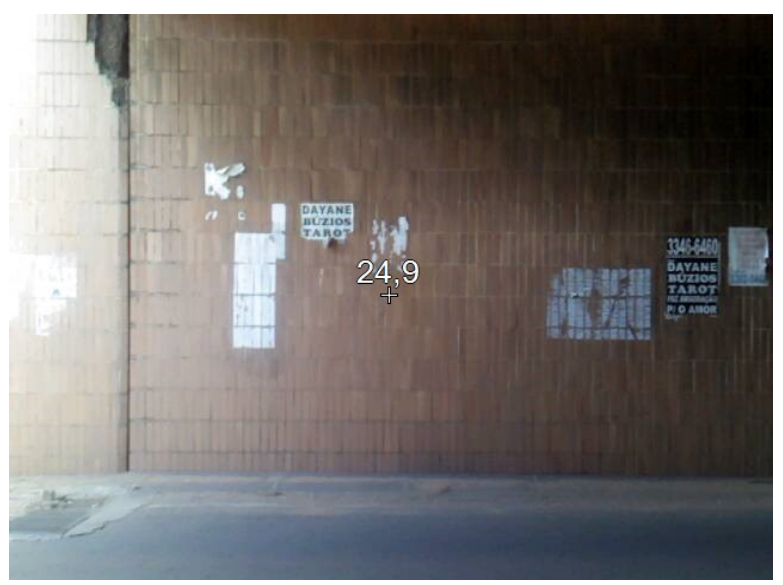

Figura 16 - Foto digital rodapé da cortina 